\title{
Adeninnucleotide und freie Aminosäuren in der Rattenleber sowie Amino- säure- und Corticosteronspiegel im Serum von Ratten nach Verbrennungen
}

\author{
Von H. KRöner, E. Meinken und W. StaiB') \\ Aus dem Pbysiologisch-chemischen Institut der Universität Dïsseldorf (Direktor: Prof. Dr. S. Hollmann)
}

(Eingegangen am 28. Januar 1966)

\begin{abstract}
Nach einer standardisierten Verbrennung an Ratten wurden zu verschiedenen Zeiten die freien Aminosäuren der Leber und des Plasmas, die Adeninnucleotide in der Leber und der Plasma-Corticosteronspiegel untersucht. Auf die Schockphase mit erhöhten Corticosteron- und Aminosäurespiegel im Plasma folgt ein leeres Intervall von etwa sechs Tagen. In der dann folgenden zweiten Phase sind bei erniedrigtem Energiequotienten ATP/ADP zunächst die Aminosäuren der Leber vermehrt, danach steigt der Plasma-Aminosäurespiegel an. Ein
\end{abstract} möglicher kausaler Zusammenhang der Befunde wird diskutiert.

The free amino acids of liver and plasma, the adenine nucleotides of the liver and the level of plasma corticosterone were studied after the administration of burns under standard conditions. The shock phase, with increased levels of corticosterone and amino acids in the plasma, was followed by a blank interval of about 6 days. The second phase then occurred, with a lowered ATP/ADP ratio, in which the amino acids of liver were increased, followed by an increase in the plasma amino acid level. The time and causal relationships of the findings are discussed and the results are compared with the findings of other authors.

Unsere zunehmenden Kenntnisse über die Pathophysiologie der Verbrennungskrankheit betreffen in erster Linie die etwa 48 Stunden dauernde Schockphase $(1,2)$. In den späteren Stadien sind zwar verschiedene pathologische Veränderungen festgestellt worden, aber ihre Bedeutung für den Krankheitsverlauf ist noch nicht gesichert. Aufgrund früherer tierexperimenteller Untersuchungen $(3,4)$ können wir isolierte Leber- oder Nierenschädigungen größeren Ausmaßes ausschließen. Die Infektion ist aber trotz moderner antibiotischer Therapie noch ein Problem (5). Zur Diskussion stehen ferner Wirkungen nicht bakterieller Toxine (6). Von den verschiedensten metabolischen Veränderungen (7) dürfte der Störung des Proteinstoffwechsels mit einer noch Wochen nach der Verbrennung bestehenden negativen Stickstoffbilanz besondere Bedeutung zukommen.

In diesem Zusammenhang erschien uns eine Mitteilung von LINDLAR und BERGER (8) interessant, die im Spätstadium einer Verbrennung eine Aminosäureanreicherung bei einer Patientin fanden. $\mathrm{Da}$ für die Proteinsynthese nicht nur die Bausteine, sondern auch Energie erforderlich ist, haben wir den Gehalt an freien Aminosäuren und an Adeninnucleotiden in der Leber untersucht. Aüfschlußreich erschien uns ferner das. Verhalten der freien Plasma-Aminosäuren sowie die Funktion der Nebennierenrinde, erfaßbar über die CorticosteronBlutspiegel (9).

\section{Methodik}

Bei männlichen Wistarratten $(250-350 \mathrm{~g})$ wurden mit der früher angegebenen Methodik (3) - 10 Sek. $250^{\circ}, 25 \%$ der Oberfläche Verbrennungen gesetzt. Für die Bestimmung der Aminosäuren blieben die Tiere 24 Stdn. vór dem Töten nüchtern, erhielten aber Wasser ad libitum. In leichter Athernarkose wurde mittels Herzpunktion Blut gewonnen, das wir mit einem Tropfen Liquemin versetzten und $10 \mathrm{Min}$. bei 3000 U./Min. zentrifugierten. Das Plasma wurde 1:10 mit 0,6N Perchlorsäure enteiweißt. Direkt nach dex Punktion entnahmen wir etwa $2 \mathrm{~g}$ Leber, homogenisierten $2 \mathrm{Min}$. lang im „MSE-Homogenisator" mit $10 \mathrm{ml}$ 0,6 N Perchloțsäure, gaben weitere $15 \mathrm{ml}$ Perchlorsäure hinzu und zentrifugierten 10 Min. bei 3000 U./Min. Die eiweißfreien Extrakte wurden mit

1) Mit einer finanziellen Beihilfe der Hohen Behörde der Europäischen Gemeinschaft für Koble und Stahl durchgeführte Forschungsarbeit.
2N KOH neutralisiext, gekühlt, der Perchlorat-Niederschlag abzentrifugiert.

Nach der Verdünnung der Leberextrakte 1:10 mit Wasser bestimmten wir die freien Aminosäuren nach MOore und SteIN (10) mit dem modifizierten Ninhydrinreagenz nach SPAKMAN, STEIN und MOore (11). Als Eichsubstanz diente Leucin der Fa. E. Merck, Darmstadt (10-30 $\mu \mathrm{g} /$ Ansatz). Um inkontrollierbare Schwankungen möglichst auszuschalten, wurde jedem Versuchstier ein gleichbehandeltes Kontrolltier ohne Verbrennung zugeordnct und die Abweichung von den Kontrollwerten in Prozent angegeben. Alle aufgeführten Werte sind Mittelwerte aus mindestens fünf Einzelwerten.

Einen Teil des gewonnenen Plasmas nahmen wir zur Bestimmung des Corticosterons nach Zenker und Bernstern (12) in der Modifikation von PFEIFFER und Mitarbeitern (9).

Für die Bestimmung der freien Adeninnucleotide entnahmen wir von Tieren in leichter Äthernarkose etwa $2 \mathrm{~g}$ Leber mittels Frierstop, zerkleinerten das Gewebe im Mörser unter flüssigem Stickstoff und gaben es anschließend unter Schütteln in $20 \mathrm{ml}$ vorgewogene, gekühlte, $0,6 \mathrm{~N}$ Perchlorsäure. Nach Differenzwägung wurde zur Vervollständigung der Extraktion 2 Min. im „MSEHomogenisator" bei Eiskühlung homogenisiert, anschließend in einer Kühlzentrifuge $15 \mathrm{Min}$. bei $18000 \mathrm{U}$./Min. zentrifugiert. Wir neutralisierten den Überstand mit $2 \mathrm{~N} \mathrm{KOH}$ gegen Methylorange, stellten ihn $30 \mathrm{Min}$. in Eiswasser zur Perchloratfällung und filtrierten.

Wir bestimmten ATP mittels Hexokinase und Glukose-6-phosphatdehydrogenase nach LAMprecht und Trautschold (13), ADP und AMP mit Pyruvatkinase und Lactatdehydrogenase (Boehringer Test TC-K). Durch das zugesetzte reduzierte NAD ist ein Leerwert bedingt, der besonders bei der AMP-Bestimmung ins Gewicht fällt. Er wurde von uns eliminiert, indem wir einen zweiten Bestimmungsansatz mit doppelter Extraktmenge durchführten. Bildet man die Differenz der beiden gemessenen Extinktionen, so fällt dadurch der Reagenzienleerwert fort.

Die statistische Sicherung erfolgte mittels t-Test nach FrSCHERSTUDENT.

\section{Ergebnisse}

Die prozentuale $\mathrm{Zu}-\mathrm{bzw}$. Abnahme der Aminosäurekonzentration gegenüber entsprechenden Kontrollen ist in Abbildung 1 gegen die Zeit aufgetragen. Die absoluten Normalwerte, berechnet als Mittelwert aller Kontrollen $(n=80) \pm$ Standardabweichung betragen $0,85 \pm 0,149 \mathrm{mg} / \mathrm{ml}$ Plasma und $7,97 \pm 0,982 \mathrm{mg} / \mathrm{g}$ Leberfrischgewicht.

Kurzfristig nach der Verbrennung steigen die freien Plasma-Aminosäuren steil an. Das Maximum liegt bei 4 Stdn. mit einer Zunahme von über $50 \%$. Im Diagramm 


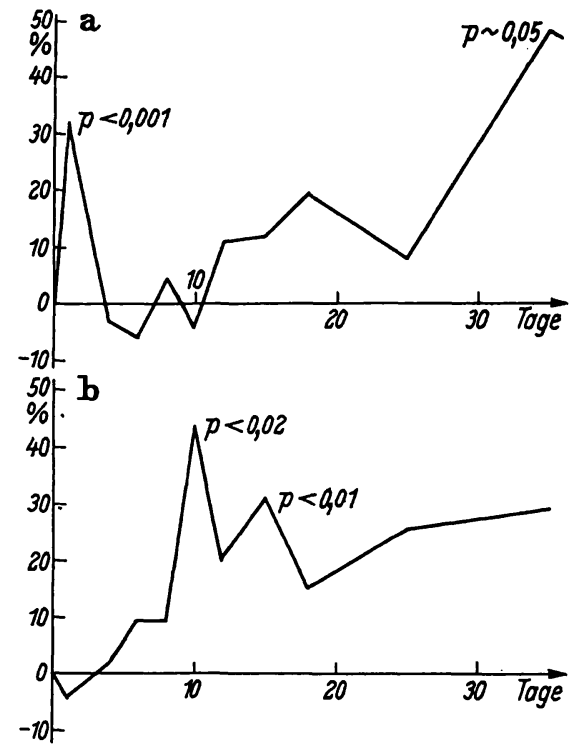

Abb. 1

Veränderung der Konzentration der Aminosäuren a) im Plasma, b) in der Leber von Ratten nach Verbrennung. Jeder Wert entspricht mindestens fünf Einzelwerten. $\mathrm{Zu}$ jedem Wert wurden mindestens 5 Kontrollen durchgeführt, deren Mittel als $100 \%$ $\mathrm{p}=$ Signifikanz der Differenz zu den Kontrollen

$1 \mathrm{a}$ ist der Wert von 24 Stdn. nach der Verbrennung eingetragen, der mit $+32 \%$ immer noch hochsignifikant $(p<0,001)$ von den Kontrollen abweicht. Einen zweiten Anstieg fanden wir vom 12. Tage an, aber erst das Maximum am 18. Tage mit $+19 \%$ unterscheidet sich signifikant von den Kontrollen. Ein dritter Anstieg am 35. Tag nach der Verbrennung auf $+48 \%$ hat eine Wahrscheinlichkeit von $\mathrm{p}=5,1 \%$. Im Gegensatz $\mathrm{zu}$ den Aminosäuren des Plasmas verändern sich die der Leber (Abb. 1b) zunächst kaum. Erst am 10. Tag findet sich eine deutliche, signifikante $(p<0,02)$ Vermehrung auf $+44 \%$. Die Konzentration der Aminosäuren in der Leber bleibt gering erhöht, aber nur das zweite Maximum am 15. Tag nach der Verbrennung unterscheidet sich noch signifikant $(p<0,01)$ mit $+31 \%$ von den Kontrollen.

Das Corticosteron im Plasma steigt kurzfristig nach der Verbrennung signifikant an. Schon nach einer Stunde liegen die Werte um $50 \%$ höher als die der Kontrollen und bleiben auch nach 4 und 8 Stunden signifikant über $50 \%$ erhöht. 24 Stdn. nach dem Trauma ist der Corticosteronspiegel wieder abgefallen und zeigt danach nur noch uncharakteristische Schwankungen geringen Ausmaßes.

Unsere Normalwerte für die Adeninnucleotide \pm Standardabweichung in der Leber betragen bei 6 Einzelwerten : ATP : $2,82 \pm 0,24$; - ADP : $0,87 \pm 0,13$; AMP : 0,32 $\pm 0,11$ jeweils $\mu \mathrm{Mol} / g$ Leberfrischgewicht. Während sich die Summe der Adeninnucleotide je Gramm Leberfrischgewicht nicht wesentlich ändert, verschiebt sich das Verhältnis ATP : ADP nach einer Verbrennung im beobachteten Zeitraum. In Abbildung 2 sind die prozentualen Anteile der drei Adeninnucleotide und ihre zeitlichen Veränderungen aufgezeichnet. Man erkennt eine Zunahme des ADP-Anteils von $22 \%$ als Ausgangswert auf $27 \%$ am 10 . Tag und $28 \%$ am 15 . Tag.

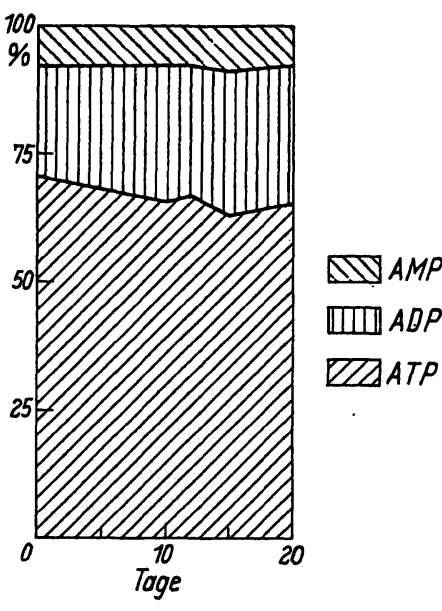

Abb. 2

Adeninnucleotide in der Leber nach Verbrennung.

Die Summe der Adeninnucleotide wurde gleich $100 \%$ gesetzt. (Mittelwerte von je 6 Versuchen)

Die Zunahme ist hochsignifikant $(p<0,001)$ und exfolgt auf Kosten einer relativen Verminderung des ATP von $70 \%$ auf $65 \%$ respektive $63 \%$. Der Anteil des AMP bleibt mit etwa 8\% konstant. Am 20. Tag nảch der Verbrennung ist $z$ war das Verhältnis ATP : ADP noch nicht wieder normal, jedoch nimmt die Streuung erheblich zu, und die Abweichung vom Normalwert ist nicht mehr signifikant.

\section{Diskussion}

Ordnet man die gefundenen pathologischen Veränderungen in ihrer zeitlichen Reihenfolge einander $z u$, so ergibt sich folgendes Bild:

Eine Stunde nach der Verbrennung steigt der Corticosteronspiegel im Blut an, nur wenig später folgt ein Anstieg der freien Aminosäuren. Zwischen beiden Veränderungen dürfte ein kausaler Zusammenhang bestehen (14). Daneben ist zu erwähnen, daß ENGEL und Mitarbeiter (15) im Blutungsschock auch bei nebennierenlosen Tieren einen Anstieg der Plasma-Aminosäuren feststellen konnten. Ferner könnte ein Teil der Aminosäuren aus dem hitzegeschädigten Gewebe stammen. Ob darüber hinaus noch eine gestörte Leberfunktion zur Erklärung des Anstiegs der Plasma-Aminosäuren (16) herangezogen werden muß, erscheint zweifelhaft, da der Gehalt der Leber an freien Aminosäuren sich in der Schockphase nicht verändert. An dieses Frühstadium der Verbrennungskrankheit schließt sich ein bei unseren Untersuchungen leeres Intervall von etwa 6 Tagen an. Erst dann erfolgt ein erheblicher Anstieg der Aminosäuren in der Leber bei einem erniedrigten Energiequotienten ATP/ADP. Die Aminosäuren im Plasma sind zu diesem Zeitpunkt sicher nicht verändert. Auch am Ende der zweiten Woche, wo die Aminosäuren der Leber nochmals signifikant erhöht gefunden wurden, bei gleichzeitig erniedrigten Energiequotienten in der Leber, ist der Plasma-Aminosäurespiegel nur mäßig und nicht signifikant erhöht. Sein Maximum erreicht dieser erst zu einem Zeitpunkt, da die Veränderungen in der Leber sich schon wieder weitgehend normalisiert haben. Der Corticosteronspiegel bleibt während dieser zweiten Phase normal. 
Allein aufgrund der zeitlichen Reihenfolge der erhobenen Befunde läßt sich erkennen, $\mathrm{da} B$ es neben der ersten schockbedingten Störung des Aminosäure-Eiweißstoffwechsels eine zweite, anders geartete Störung dieses Stoffwechsels gibt. Nimmt man für die von uns während der zweiten Phase der Verbrennungskrankheit erhobenen Befunde nicht nur einen rein zeitlichen $\mathrm{Zu}$ sammenhang an, so ist unseres Erachtens die einfachste Erklärung die, daß der erniedrigte Energiequotient die Ursache für die Störung des Aminosäure-Protein-Stoffwechsels ist. Ein erster Hinweis für die primäre Bedeutung des Energiestoffwechsels kommt von der Morphologie. Der häufigste p̈athologisch-anatomische Befund der Leber nach Verbrennung ist eine trübe Schwellung (7). Sie ist bedingt durch eine Quellung der
Mitochondrien und damit morphologisches Substrat der Energiestoffwechselstörung (17). Eine weitere Bestätigung erfährt unsere Annahme durch die Befunde von KoEHN (18). Er fand als Ausdruck einer Zerstörung von Enzymen der Atmungskette eine Abnahme der Flavinnucleotide in der Leber nach Verbrennung. Pathogenetische Zusammenhänge zwischen den beobachteten Stoffwechselstörungen, nicht bakteriellen Toxinen und häufig therapieresistenten Infekten sind vorstellbar, aber bisher ungeklärt.

Fräulein G. JungebLodt danken wir für ihre fleißige und gewissenhafte Mitarbeit bei der Durchführung der Versuche. Ein weiterer Dank gilt Herrn Gewerbeobermedizinalrat Dr. TFr. PETERs für seine wohlwollende Unterstützung unseres Forschungsprogramms.

\title{
Literatur
}

1. Müller, F. E., Med. Klinik 60, 636 (1965). - 2. RehN, J. und L. Kosıowskr, Praktikum der Verbrennungskrankheit, Vorträge aus der praktischen Chirurgie, 57. Heft Ferdinand Enke, Verlag, Stuttgart (1960). - 3. RüENAuver, R., H. KröNER und W. Staib, Hoppe-Seyler's Z. physiol. Chem. 331, 143 (1963). - 4. KRÖNER, H. und R. Rürenauver, Hoppe-Seylex's Z. physiol. Chem. 336, 227 (1964). - 5. Arturson, G., Acta chir. Scand. 128, 25 (1964). 6. ReHN, J., Langenbeck's Arch. Klin. Chir. 290, 466 (1959). 7. AllgöWER, M. und J. Siggerist, Verbrennungen, SpringerVerlag, Berlin-Göttingen-Heidelberg (1957). - 8. LIndLAR, F. und H. Berger, Schweiz. med. Wschr. 92, 110 (1962). - 9. Zenker, N. und D. E. Bernstein, J. biol. Chemistry 231, 695 (1958). - 10. Moore, S. und W. H. Stein, J. biol. Chemistry 176, 367 (1948). - 11. Spackman, D. H., W. H. Stein und S. Moore. Analytic. Chem. 30, 1190 (1958). - 12. Pfeiffer, E. F., W. E. Vaubel, K. Retiene, D. Berg und H. Ditschuneit, Klin.
Wschr. 38, 980 (1960). - 13. Lamprecht, W. und I. TrautSChold, In Methoden der enzymatischen Analyse. Hrsg. H. U. Bergmeyer, Verlag Chemie GmbH, Weinheim/Bergstr. (1962). - 14. FRIEDberg, F. und D. M. Greenberg, J. biol. Chemistry 168, 405 (1947); Bondy, P. K., Endocrinology 45, 605 (1949); INGLE, D. J., M. C. Prestrud und J. E. Nezamis, Proc. Soc. exp. Biol. Med. 75, 801 (1950); Kaplan, S. A. und C. S. Nagareda Shimizu, Endocrinology 72, 267 (1963). - 15. ENGEL, F. L., M. G. Wintou und C. N. H. Long, J. Exper. Med. 77, 397 (1943). - 16. ENGEL, F. L., Ann. N. Y. Acad. Sci. 55, 381 (1952); Metabolic aspects of hemorrhagic and traumatic shock. 2. Conf. on Shock and Circulatory Homeostasis. Josiak Macy jr. Foundation, New York 1952. 17. Büchner, F., Allgemeine Pathologie, 2. Aufl. Urban \& Schwarzenberg, München-Berlin (1956). - 18. КовнN, C. J., Ann. Surg. 158, 933 (1963).

Professor Dr. W. Staib, 4 Düsseldorf, Witzelstraße 111

\section{Zur Bestimmung von Ketosteroiden mit 2,4-Dinitrophenylhydrazin}

\author{
I. Mitteilung: Beschreibung der Methode')
}

Von L. Tretber und G. W. Oertel (Aus der Abteilung für Experimentelle Endokrinologie, Institut für Hygiene und Mikrobiologie,
Direktor: Prof. Dr.Dr.W..Zimmermann, Universität des Saarlandes, Homburg/Saar)

(Eingegangen am 20. Juni 1966)

Für die qualitative und quantitative Bestimmung von Ketosteroiden eignet sich ihre Reaktion mit 2,4-Dinitrophenylhydrazin in abs. Benzol/Trichloressigsäure. Die gebildeten 2,4-Dinitrophenylhydrazone von $C_{21^{-}}, C_{19^{-}}$und $C_{18}$-steroiden mit Ketogruppen u. a. in C-3, C-17 oder C-20 werden vermittels Dünnschichtchromatographie abgetrennt und photometriert. Hierbei hängen Wellenlänge des Absorptionsmaximums $(360-390 \mathrm{~m} \mu)$ und molarer Extinktionskoeffizient $(\varepsilon=22000-52000)$ von Zahl und Stellung der Ketogruppen ab. Die vorliegende Methode gestattet die Erfassung von 0,1 $\mu \mathrm{g}$ Monoketosteroid in $1 \mathrm{~m} l$ Endlösung mit ausreichender Richtigkeit und Genauigkeit.

The reaction of oxo-steroids with 2.4-dinitrophenylhydrazine in absolute benzene/trichloracetic acid may be employed for their qualitative and quantitative determination. The 2.4-dinitrophenylhydrazones of $\mathrm{C}_{21^{-}}, \mathrm{C}_{19^{-}}$, and $\mathrm{C}_{18^{-}}$-steroids with oxo groups in position $\mathrm{C}-3, \mathrm{C}-17$ or $\mathrm{C}-20$, are separated by thin layer chromatography and measured photometrically. The absorption maximum $(360-390 \mathrm{~m} \mu)$ and the molar extinction coefficient $(\varepsilon=22,000-52,000)$ depend on the number and position of the oxo groups. With the present assay, it is possible to determine $0.1 \mu \mathrm{g}$ of a mono-oxo-steroid in $1 \mathrm{ml}$ of final solution with sufficient accuracy and precision.

Obgleich sich die Zimmermann-Reaktion als Nachweis von 17-Ketosteroiden in qualitativen und quantitativen Untersuchungen hinlänglich bewährt hat, so fehlt ihr doch die Empfindlichkeit, wie sie z. B. zur Bestimmung vorgenannter $\mathrm{C}_{19}$-steroide in geringen Plasmavolumina erforderlich ist. Es lag infolgedessen nahe, anstelle der

1) Vorliegende Arbeit wurde mit Unterstützung der Deutschen Forschungsgemeinschaft, Bad Godesberg, durchgeführt. zwar spezifischen Zimmermann-Reaktion die weitaus empfindlichere Umsetzung von Ketosteroiden mit 2,4-Dinitrophenylhydrazin zur quantitativen Erfassung derartiger Verbindungen heranzuziehen, zumal die Beständigkeit der gebildeten Derivate und ihre intensive Farbe sowohl die Handhabung, wie auch eine etwaige chromatographische Isolierung erleichtern sollte. Aufbauend auf den grundlegenden Untersuchungen von 